Pis'ma v ZhETF, vol. 88, iss. 9, pp. $698-701$ (2008)

\title{
Electron energy level statistics in graphene quantum dots
}

\author{
H. De Raedt* and M. I. Katsnelson ${ }^{+1)}$ \\ * Department of Applied Physics, Zernike Institute for Advanced Materials \\ University of Groningen, Nijenborgh 4, NL-9747 AG Groningen, The Netherlands \\ ${ }^{+}$Institute of Molecules and Materials, Radboud University of Nijmegen \\ NL-6525 ED Nijmegen, The Netherlands \\ Submitted 12 August 2008 \\ Resubmitted 12 September 2008
}

\begin{abstract}
Motivated by recent experimental observations of size quantization of electron energy levels in graphene quantum dots [7] we investigate the level statistics in the simplest tight-binding model for different dot shapes by computer simulation. The results are in a reasonable agreement with the experiment which confirms qualitatively interpretation of observed level statistics in terms of "Dirac billiards" without taking into account many-body effects. It is shown that edge effects are in general sufficient to produce the observed level distribution and that even strong bulk disorder does not change the results drastically.
\end{abstract}

'PACS: 73.22.Dj, 81.05.Uw, 05.45.Mt

Graphene attracts enormous attention now, due to 'its interest both for fundamental physics, such as opportunities to simulate in condensed matter experiments subtle quantum relativistic effects and for potential applications, as a planar, high-mobility material for "post"silicon" electronics [1, 2, 3, 4, 5. Graphene-based nan'odevices are subjects of especial interest. Recently, size quantization effects have been observed in graphene nanoribbons [6] and quantum dots (QD) [7]. It was demonstrated that for QD smaller than $100 \mathrm{~nm}$ the 'electron energy spectrum is essentially irregular demonstrating a "chaotic" behavior. The latter can be dis"cussed in terms of random matrix theory for a single"particle problem 8, 9, 10. However, in general, because 'of interplay of size quantization and Coulomb blockade 'correlation effects can be important for QD [11; more"over, in some limiting cases the "chaotic" energy spec"trum can be described even purely classically, in terms of only Coulomb energies [12]. Therefore, to understand -properly the experimental data [7] some theoretical efforts are necessary.

In this Letter we present the results of straightforward computer simulations of level statistics in graphene QD, adopting the simplest one-electron picture, up to 16000 sites (which is comparable to the sizes of smallest QD investigated experimentally). It turns out that already this approach does allow to reproduce, in a semi-quantitative way, the observed energy distribution.

As the model Hamiltonian, we take the simplest nearest-neighbor tight-binding model on a hexagonal

\footnotetext{
1) e-mail: M.Katsnelson@science.ru.nl
}

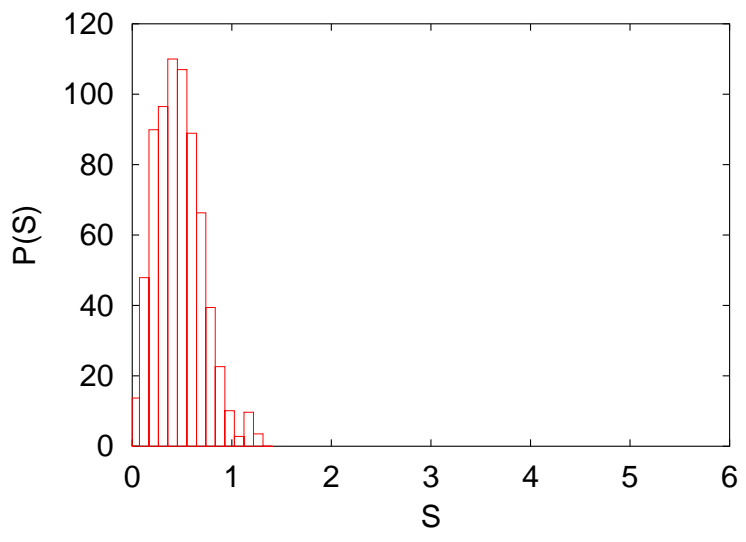

Fig. 1 (Color online) Level-spacing distribution $P(S)$ as obtained from experiment [7] for the case of $40 \mathrm{~nm}$ QD (The raw experimental data are by courtesy of $K$. Novoselov and A. Geim).

lattice as introduced in Ref. [13, enclosed in some geometrical shape, such as a circle, triangle, etc. The Hamiltonian reads

$$
\begin{aligned}
H= & -t \sum_{\langle i, j\rangle} \sum_{\sigma=\uparrow, \downarrow}\left(a_{i, \sigma}^{\dagger} b_{j, \sigma}+b_{j, \sigma}^{\dagger} a_{i, \sigma}\right) \\
& +\sum_{i \in \mathcal{B}} \sum_{\sigma=\uparrow, \downarrow} V_{i} a_{i, \sigma}^{\dagger} a_{i, \sigma}+\sum_{j \in \mathcal{B}} \sum_{\sigma=\uparrow, \downarrow} V_{j} b_{j, \sigma}^{\dagger} b_{j, \sigma} \\
& +\sum_{i} \sum_{\sigma=\uparrow, \downarrow} v_{i} a_{i, \sigma}^{\dagger} a_{i, \sigma}+\sum_{j} \sum_{\sigma=\uparrow, \downarrow} v_{j} b_{j, \sigma}^{\dagger} b_{j, \sigma},
\end{aligned}
$$

where $t$ is the nearest-neighbor hopping energy, $a_{i, \sigma}^{\dagger}$ $\left(a_{i, \sigma}\right)$ creates (annihilates) an electron with spin $\sigma$ on 
one of the sub-lattices of the hexagonal lattice and $b_{j, \sigma}^{\dagger}$ $\left(b_{j, \sigma}\right)$ creates (annihilates) an electron with spin $\sigma$ on the other sub-lattice of the hexagonal lattice. In the expression for the hopping term, the indices $i$ and $j$ run over all nearest neighbors only.

Following Ref. [14, we also consider the effects of a staggered on-site potentials $V_{i}$ and $V_{j}$ that alternate as we move along the boundary $\mathcal{B}$ of the lattice; as shown in Ref. 14, the continuum limit with a very large $V_{i}$ and $V_{j}$ corresponds to the "infinite mass" boundary condition [8. The physical origin of this potential can be related to, for instance, the magnetic moments at the zigzag edges [15, 16]. Of course, in a simulation we can easily study the effect of $V_{i}$ by considering the cases $V_{i}=V_{j}=0$ and $V_{i} \neq 0$ and $V_{j} \neq 0$. Optionally, to study the effect of disorder, we use uniform pseudorandom numbers to choose the hopping integrals from the interval $[t-\delta, t+\delta]$ and/or we add on-site potentials $v_{i}$ and $v_{j}$ in the range $[-v, v]$.

The geometrical shape "cuts out" a piece of the hexagonal lattice such that armchair and zigzag parts of the boundary appear (for a general discussion of boundary conditions for the tight-binding model of graphene, see Ref. [14]). Furthermore, we study the effect of disorder that results from removing hexagons from the regular hexagonal layer.

The eigenvalues of Hamiltonian Eq. (11) are obtained by exact numerical diagonalization. As we are interested in the part of the spectrum that, in the continuum limit corresponds to the spectrum of the Dirac equation, we limit the search for eigenvalues to the interval $[-0.4 t, 0.4 t]$. In our numerical work, we adopt units such that $\hbar=1$ and we set the hopping integral $t=1$.

For reference, in Fig. 1, we show the experimental results of the level spacing distribution for a graphene dot of $40 \mathrm{~nm}$ diameter [7]. The dimensionless level spacing $S$ is defined as the energy difference $\Delta E_{i}=E_{i}-E_{i-1}$ between successive levels, divided by the average $\left\langle\Delta E_{i}\right\rangle$ of the energy differences between successive levels. The number $P(S)$ gives the number of energy difference for which $S-\Delta / 2<\Delta E_{i} /\left\langle\Delta E_{i}\right\rangle \leq S+\Delta / 2$, where $\Delta$ is the bin size of the histogram. The experimental data for the distribution have been shifted by $0.02 \mathrm{~V}$ to remove an ambiguity in the definition of $S=0$.

For comparison, Fig. 2 shows the level distribution for a hexagonal lattice with periodic boundary conditions, a lattice that has not been cut-out using some geometrical shape. It is clear that its spectrum does not resemble the one observed experimentally.

Next, we consider the case of a circular dot. In the continuum approximation with the infinite mass boundary conditions 8 this case is special, with separable

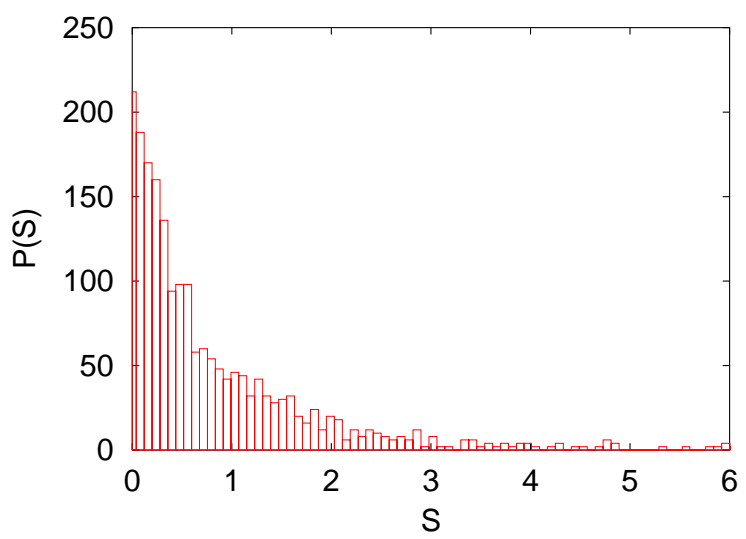

Fig. 2 (Color online) Level-spacing distribution $P(S)$ for a hexagonal lattice with periodic boundary conditions, using 170 different values for both $k_{x}$ and $k_{y}$.

variables and a regular energy spectrum. In reality, although we use a circle to cut out from the infinite lattice, the boundary of this lattice is irregular: There are short and long pieces of the armchair and zigzag boundaries. This kind of irregularities destroy the circular symmetry of the the geometrical shape completely.

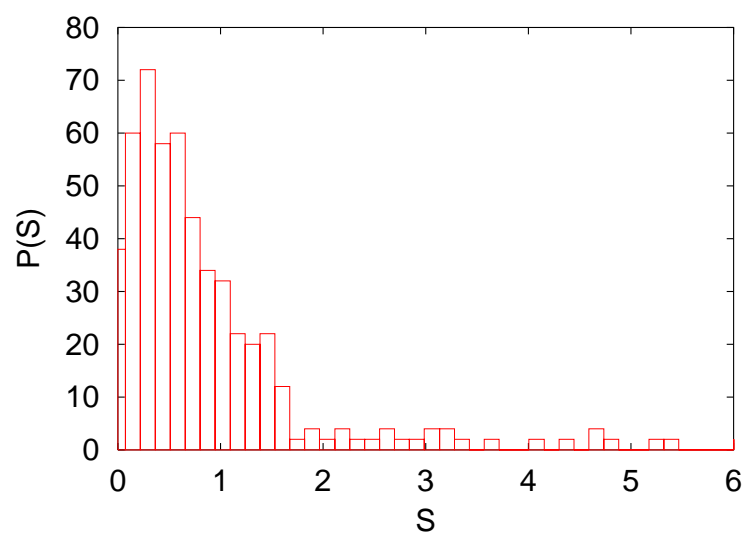

Fig. 3 (Color online) Level-spacing distribution $P(S)$ for a hexagonal lattice bounded by a circle. The number of hexagons inside the circle is 8202 .

In Figs. 3 and 4 , we depict the results for $P(S)$ and the density of states $\operatorname{DOS}(E)$ for the case of the perfect hexagonal lattice, bounded by a circle. Although there is a clear background linear dependence of the $\operatorname{DOS}(E)$ on $E$, it is also clear that there are fluctuations due to size quantization. In the DOS, the peak around zero energy is due to the existence of the zigzag edge states, as expected for a generic boundary [14]. Comparing Fig. 3 


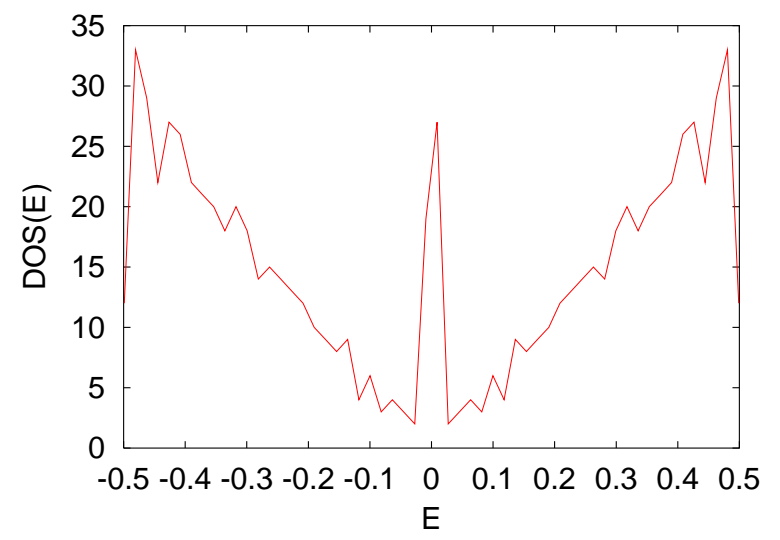

Fig. 4 (Color online) Density of states $\operatorname{DOS}(E)$ as a function of the energy $E$ for a hexagonal lattice confined to a circle. The number of hexagons inside the circle is 8202 .

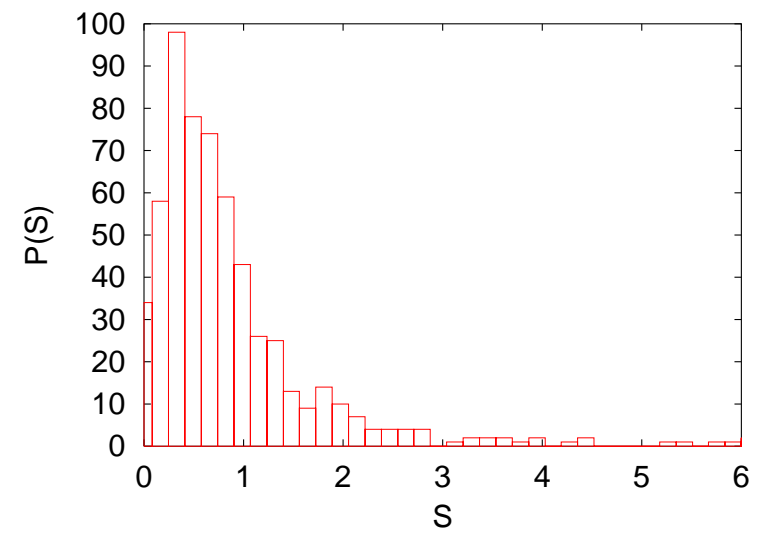

Fig. 5 (Color online) Same as Fig. 3 except that the staggered potential at the boundary edges $V=100$, the hopping integrals fluctuate by maximum $20 \%$ ( $\delta=$ 0.2 ) and the on-site potentials fluctuate in the range $[-0.2,0.2]$ The number of hexagons inside the circle is 8484 .

with the experimental result Fig. 1 we conclude that there is little resemblance.

In contrast, by introducing various forms of disorder to the same system, we find semi-quantitative agreement, as shown in Figs. 5 and 6 . Our numerical experiments (not all results shown) suggest that the presence of an alternating boundary potential can change the qualitative features of $P(S)$ significantly. Including various forms of disorder $(\delta \neq 0, v \neq 0$ and remove some hexagons), the level distribution $P(S)$ (see Fig. 6) looks similar to the experimental result (see Fig. 11). Of course, we cannot expect quantitative agreement: The number of items in the experimental data is about 60

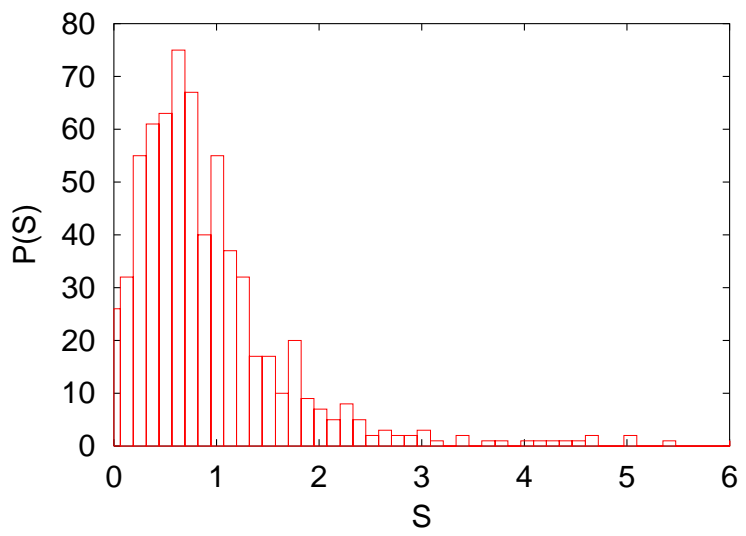

Fig. 6 (Color online) Same as Fig. 3 except that the potential at the boundary edges $V=10$, about $20 \%$ of the hexagons have been removed, the hopping integrals fluctuate by maximum $20 \%(\delta=0.2)$ and the on-site potentials fluctuate in the range $[-0.2,0.2]$ The number of hexagons inside the circle is 6540 .

while in the numerical simulations there are about 600 eigenvalues that contribute to $P(S)$. This may explain why the simulation results for $P(S)$ show a more extended tail than the experimental result for $P(S)$.

Finally, to study the effect of the shape of the boundary on the level spacing distribution, we have calculates $P(S)$ for various lemon-shaped billiards (results not shown) defined by $y= \pm\left(1-|x|^{d}\right)$ for $x \in[-1,1][17$. As a function of the shape parameters $d>0$, these billiards look like a square $(d=1, \infty)$ or two parabola $(d=2)$ or have some intermediate lemon-like shape. It has been shown that, as a function of $d$, the these billiard, classical as well as quantum mechanically, exhibit regular and chaotic behavior [17. If Figs. 7 and 8 , we present some typical results for a quarter lemon with $d=3.1$. Our motivation for presenting the results of a quarter lemon instead of the complete lemon is to show a case in which there are long stretches of armchair and zigzag boundaries (the edges along the $x$ and $y$ axis) and an irregular boundary (the curved edge). Although on purpose, we did not include the alternating potential at the boundary sites, the disorder resulting from the irregular shape together with the fluctuating on-site potential $v$ seem to be sufficient to observe a $P(S)$ that is similar to the distribution observed experimentally. Comparing Figs. 7 and 8 one can see that the level statistics is not too sensitive to the bulk disorder. There is only randomness due to boundaries themselves in one case (Fig. 7) and a random potential $v$ is introduced, additionally, in the other one (Fig. 8), but the results look very similar. 


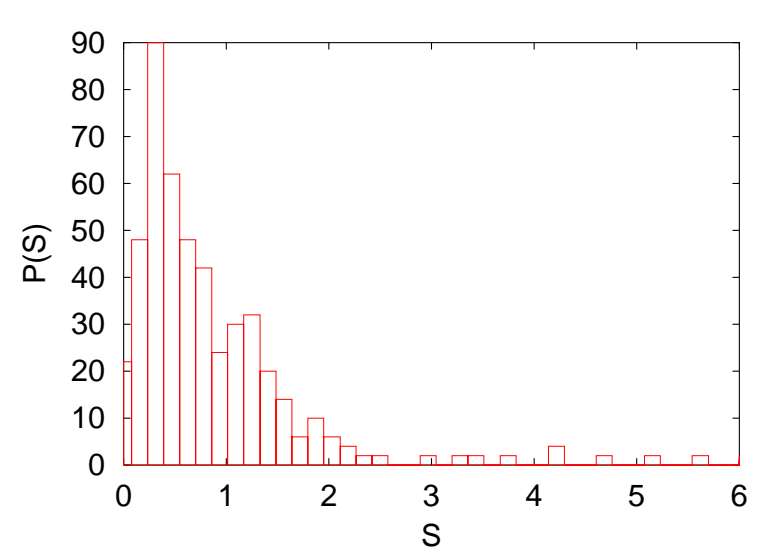

Fig. 7 (Color online) Level-spacing distribution $P(S)$ as a function of the level spacing $S$ for a hexagonal lattice bounded by a quarter of a lemon-shaped billiard for $V=\delta=v=0$. Parameter of the lemon: $d=3.1$ ( $d=1$ is half of a triangle, $d=2$ is a half of parabola). The number of hexagons inside the billiard is 7861 .

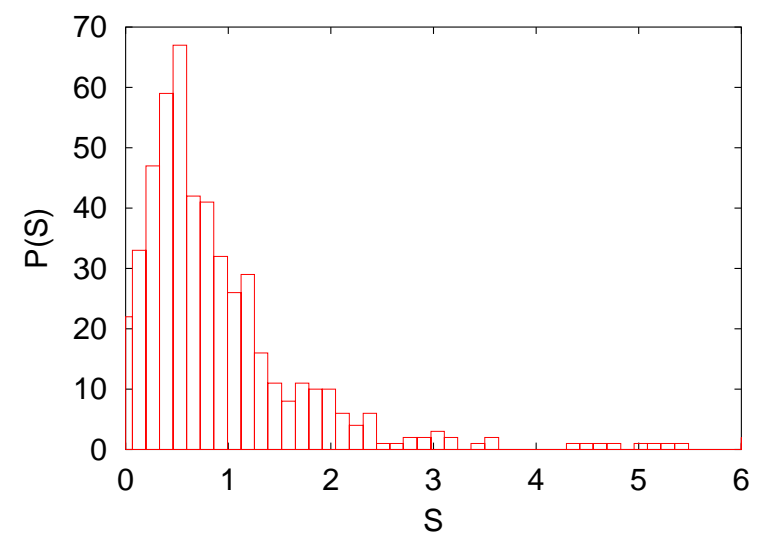

Fig. 8 (Color online) Same as Fig. 7 except that $v$ is chosen at random from $[-0.2,0.2]$.

To conclude, it seems that disorder due to randomness of the edges is, in principle, enough to reproduce the experimentally observed level distribution which makes the term "chaotic Dirac billiard" quite reasonable. One may need the local on-site disorder and some disorder in the hopping integrals to get semi-qualitative agreement with experiment but, on the other hand, the simulated systems do not have the same shape and are not as large as the experimental ones so a complete quantitative agreement is, anyway, hard to expect.

It is worth mentioning that the continuum approximation may be a bit dangerous when discussing the level statistics in graphene QDs. In our simulations, we do not see any essential differences between "regular" (cir- cular) and "irregular" (lemon-shaped) billiards. Even for tens of thousand sites the edge is essentially irregular with staggered armchair and zigzag pieces. Therefore, even for circular quantum dot we may have a "chaotic" energy level distribution.

We are thankful to Andre Geim and Kostya Novoselov for helpful discussions and for providing us with the original experimental data used in Fig. 1 and to Boris Shklovskii for illuminating discussions. The work was supported by the Stichting voor Fundamenteel Onderzoek der Materie (FOM) (the Netherlands)

1. A.K. Geim and K.S. Novoselov, Nature Mater. 6, 183 (2007).

2. M.I. Katsnelson, Mater. Today 10, 20 (2007).

3. S. Das Sarma, A.K. Geim, P. Kim, and A.H. MacDonald (Editors), Special Issue of Solid State Commun. 143, 1-125 (2007).

4. A.H. Castro Neto, F. Guinea, N.M.R. Peres, K.S. Novoselov, and A.K. Geim, arXiv:0709.1163 (to appear in Rev. Mod. Phys.).

5. P. Avouris, Z. Chen, and V. Perebeinos, Nature Nanotech. 2, 605 (2007).

6. M.Y. Han, B. Özyilmaz, Y. Zhang, and P. Kim, Phys. Rev. Lett. 98, 206805 (2007).

7. L.A. Ponomarenko, F. Schedin, M.I. Katsnelson, R. Yang, E.W. Hill, K.S. Novoselov, and A.K. Geim, Science 320, 356 (2008).

8. M.V. Berry and R.J. Mondragon, Proc. R. Soc. (London) A 412, 53 (1987).

9. T. Guhr, A. Müller-Groeling, and H.A. Weinedmüller, Phys. Rep. 299, 189 (1998).

10. C.W.J. Beenakker, Rev. Mod. Phys. 69, 731 (1997).

11. R. Shankar, Rev. Mod. Phys. 80, 379 (2008).

12. A.A. Koulakov, F.G. Pikus and B.I. Shklovskii, Phys. Rev. B 55, 9223 (1997).

13. P.R. Wallace, Phys. Rev. 71, 622 (1947).

14. A.R. Akhmerov and C.W.J. Beenakker (2007), arXiv:0710.2723.

15. Y.-W. Son, M.L. Cohen, and S. Louie, Nature (London) 444, 347 (2006).

16. O.V. Yazyev and M.I. Katsnelson, Phys. Rev. Lett. 100, 047209 (2008).

17. V. Lopac, I. Mrkonjić, and D. Radić, Phys. Rev. E 59, 303 (1999). 